\title{
Factors Associated with Cause-Specific Death in Russia. Data from Longitudinal Prospective Study 1977-2001
}

\author{
Svetlana A. Shalnova, Anna V. Kapustina, Alexander D. Deev, Yulia A. Balanova* \\ National Medical Research Center for Preventive Medicine \\ Petroverigsky per. 10, Moscow, 101990 Russia
}

\begin{abstract}
Aim. To evaluate the associations between main risk factors (RF) with cause-specific death in cohorts of Russian men and women.
Material and methods. Data of a number of crossectional studies conducted in different years by unified base protocol had become the subjects for the study. A total of 12,497 men and 5,039 women aged 35-74 years, residents of Moscow and S-Petersburg (former Leningrad) cities at the moment of examination, were enrolled into the study. We analyzed 17 previously selected risk factors and their associations with cause-specific mortalities: coronary heart disease (CHD), stroke, cardiovascular diseases (CVD), non-CVD, all causes. A total of 10,650 deaths were registered: 8,726 in men (for 10 years) and 1,924 - in women (for 20 years).

Results. Men died more often from all the examined causes except for stroke, what was more typical to women. Mortality in men was associated with significantly larger number of RF than in women and correlations were stronger. In particular, smoking (hazard ratio [HR] 2.25; $95 \%$ confidence interval $[95 \% \mathrm{Cl}] 1.75-2.89 ; p=0.0001)$, high blood pressure ( $\mathrm{HR} 1.78 ; 95 \% \mathrm{Cl} 1.43-2.22 ; \mathrm{p}=0.0001)$ and history of $\mathrm{CHD}(\mathrm{HR} 3.23 ; 95 \% \mathrm{Cl} 2.71-$ 3.84; $p=0.0001$ ) significantly increased CHD-related mortality in the men's cohort but were much less significant for women. The total cholesterol level demonstrated significance in men but was not even selected in the model for women. The main RF for stroke-related mortality were smoking, high blood pressure and atrial fibrillation, while for non-cardiovascular mortality there was only one common factor - smoking. Factors associated with CVD and all-cause mortality were almost the same because CVD cover more than half in the all-cause mortality, however a larger number of predictors were reported in men.

Conclusion. The data obtained indicate: 1) considerably larger number of unfavorable risk factors in the men's cohort, which significantly increased risk for death from any cause; 2) statistically more pronounced relation between risk factors and mortality rates in men as compared to women, especially note that mortality rates were followed up for 10 years in men and 20 years in women. It is obvious that successful prevention focused on risk factors must be gender-based.
\end{abstract}

Keywords: cause-specific mortality, coronary heart disease, stroke, cardiovascular disease, risk factors.

For citation: Shalnova S.A., Kapustina A.V., Deev A.D., Balanova Y.A. Factors Associated with Cause-Specific Death in Russia. Data from Longitudinal Prospective Study 1977-2001. Rational Pharmacotherapy in Cardiology 2019;15(1):4-16. DOI:10.20996/1819-6446-2019-15-1-4-16

\author{
Факторы, ассоциированные с основными причинами смерти в России. Данные многолетнего проспективного \\ исследования 1977-2001 гг. \\ Светлана Анатольевна Шальнова, Анна Владимировна Капустина, Александр Дмитриевич Деев, Юлия Андреевна Баланова* \\ Национальный медицинский исследовательский центр профилактической медицины \\ Россия, 101990, Москва, Петроверигский пер., 10
}

\begin{abstract}
Цель. Изучить влияния основных факторов риска на смертность от различных причин в когортах российских мужчин и женщин.
Материал и методы. Материалом послужили результаты исследований, проведенных в разные годы по единому протоколу. Всего в анализ включено 12497 мужчин и 5039 женщин 35-74 лет, проживающих на момент обследования в гг. Москве и Ленинграде. Анализировались 17 предварительно отобранных факторов риска (ФР) и их ассоциации со смертностью от различных причин: ишемическая болезнь сердца (ИБС), инсульт, сердечно-сосудистые заболевания (СС3), не ССЗ и все причины. За время наблюдения за смертностью зарегистрировано всего 10650 смертей, 8726 у мужчин (за 10 лет) и 1924 у женщин (за 20 лет).

Результаты. Мужчины умирают статистически значимо чаще от всех изучаемых причин, кроме инсульта, смерть от которого более характерна для женщин. Смертность у мужчин определяется значительно большим числом ФР, чем у женщин, и эта зависимость выражена сильнее. Так, курение (отношение рисков [ОР] 2,25; $95 \%$ доверительный интервал [95\%ДИ] 1,75-2,89; р=0,0001), высокое артериальное давление (ОР 1,78; 95\%ДИ 1,43-2,22; p=0,0001) и ИБС в анамнезе (ОР 3,23; 95\%ДИ 2,71-3,84; p=0,0001) значительно увеличивают смертность от ИБС в мужской когорте, но менее значимы для женщин. Уровень общего холестерина был значимым для мужчин, но не для женщин. Для обоих полов общими ФР для смертности от инсульта были курение, высокое АД и фибрилляция предсердий, а для не сердечно-сосудистых причин общим было только курение. Факторы, формирующие смертность от ССЗ и всех причин, практически одни и те же в силу существенной доли ССЗ в структуре общей смертности, однако и в этом случае у мужчин регистрируется больше прогностически неблагоприятных факторов.

Заключение. Полученные данные свидетельствуют: 1) о значительно большем количестве прогностически неблагоприятных факторов риска у мужчин, которые существенно увеличивают в мужской когорте риск смертности по любой причине, кроме инсульта; 2) о статистически значимо более выраженной силе связи между факторами и смертностью у мужчин по сравнению с женщинами, особенно, если учитывать, что смертность наблюдалась среди мужчин в течение 10 лет, а среди женщин - 20 лет. Становится очевидным, что успешная профилактика, направленная на факторы риска, должна быть гендерной.
\end{abstract}

Ключевые слова: смертность от всех причин, ишемическая болезнь сердца, инсульт, сердечно-сосудистые заболевания, факторы риска.

Для цитирования: Шальнова С.А., Капустина А.В., Деев А.Д., Баланова Ю.А. Факторы, ассоциированные с основными причинами смерти в России. Данные многолетнего проспективного исследования 1977-2001 гг. Рациональная Фармакотерапия в Кардиологии 2019;15(1): 4-16. DOI:10.20996/1819-6446-2019-15-1-4-16

Received / Поступила: 01.11.2018

Accepted / Принята в печать: 21.01.2019

*Corresponding Author (Автор, ответственный за переписку): jbalanova@gnicpm.ru 
Significant decrease in mortality rate was observed in Russia over recent years, although it is still one of the highest in Europe [1]. The role of risk factors in cardiovascular morbidity and mortality is well known. S. Yusuf et al. had demonstrated, risk factors have no borders being actual both in low- and high-income countries, in America as well as in Europe and Australia [2]. From the other side cardiovascular disease (CVD) risk factors prevalence is not the same in different countries and their influence on population may differ.

Attempts to find reasons for such high mortality rates in Russia have been made during a long time, but were not enough sufficient $[1,3,4]$. Until recently alcohol consumption and smoking were commonly believed to be the principal risk factors influencing mortality rates in Russia while other factors considered less significant $[5,6]$. That is why estimation of different factors and biomarkers which can potentially participate in increase of mortality rates in the Russian population is of great importance.

The aim of the study was to evaluate the associations between main well-known risk factors with causespecific death in cohorts of Russian men and women examined in 1975-2001.

\section{Material and methods}

The data came from representative samples of 7 crossectional surveys, included Russian part of Lipid Research Clinics Study and Moscow MONICA Project which were conducted from 1975 to 2001 by unified core protocol. All the surveys were carried out in Moscow and St. Petersburg (former Leningrad). The response rate exceeded $75 \%$. Mortality follow up data were also collected. The study design is observational prospective cohort study.

A total of 12,497 men and 5,039 women aged 35-74 years, residents of Moscow and Leningrad cities at the moment of the study, were enrolled into the analysis.

The protocol of examination included information of social and demographic characteristics, risk factors, medical history data. All the examined persons were twice measured blood pressure (BP) ( $\mathrm{mm} \mathrm{Hg}$ ) using mercury sphygmomanometer and appropriately sized arm cuffs on the right arm, heart rate (b.p.m.) measured on the a. radialis, the mean of two readings was used for analysis. Weight and height were measured with a calibrated medical scale, and without shoes or heavy clothes. Body mass index (BMI) was calculated as the weight in kilograms divided by the height in meters squared $\left(\mathrm{kg} / \mathrm{m}^{2}\right)$. Laboratory biochemical analysis included total cholesterol (TC), high density lipoprotein cholesterol (HDL-C), triglycerides (TG) levels ( $\mathrm{mmol} / \mathrm{l}$ ) routine assessment. The ECG was registered in supine
Несмотря на существенное снижение смертности в стране в последние годы, Россия все еще остается в ряду стран с самым высоким уровнем смертности в Европе [1]. Известна роль факторов риска в заболеваемости и смертности, и, как показал S. Yusuf с соавт. [2], факторы риска определяют здоровье как в бедных, так и в богатых странах. С другой стороны, распространенность факторов риска сердечно-сосудистых заболеваний (ССЗ) в разных странах не одинакова, и их воздействие на популяцию может быть различным.

Попытки понять причины высокой смертности в России предпринимаются давно и не очень успешно, в том числе, и зарубежными исследователями $[1,3,4]$. До последнего времени считалось, что основной причиной высокой смертности в нашей стране является чрезмерное потребление алкоголя и курение, а другие факторы недооценивались $[5,6]$. Поэтому нигде, как в России, важны поиски различных показателей, биомаркеров, потенциально способных влиять на динамику смертности.

Целью настоящего исследования является изучение ассоциаций основных и, стало быть, достаточно известных, факторов риска с различными причинами смерти в когортах российских мужчин и женщин, обследованных в 1975-2001 гг.

\section{Материал и методы}

Материалом послужили представительные, обследованные по единому протоколу выборки 7 исследований, проведенных в различные годы. В каждой выборке проводилось проспективное наблюдение за смертностью. Всего в анализ было включено 12497 мужчин и 5039 женщин 35-74 лет, проживающих на момент обследования в гг. Москве и Ленинграде (ныне Санкт-Петербург). Таким образом, дизайн работы представляет собой наблюдательное проспективное когортное исследование.

Протокол обследования включал стандартный опрос, содержащий информацию о социально-демографических показателях, факторах риска, анамнестических данных. По уровню образования пациенты разделялись на 3 группы: ниже среднего, среднее, выше среднего. Из поведенческих привычек оценивали статус курения (никогда не курил, бросил, курит в настоящее время) и статус потребления алкоголя (не употреблял в течение последнего года или употреблял чрезмерно > 168 г чистого этанола в неделю для мужчин и > 84 г чистого этанола в неделю для женщин).

Всем обследованным дважды измерялось артериальное давление (АД [мм рт.ст.]) и частота сердечных сокращений (ЧСС [уд/мин]) на a.radialis, в анализ включалось среднее из двух измерений. Анализировалось повышенное АДश140/90 мм рт.ст. и высокое АДश160/95 мм рт.ст. Высокая ЧСС регистрировалась при значениях частоты пульса >80 уд/мин. Измерялись рост, вес, рассчитывался индекс массы тела $\left(\right.$ ИМТ $=$ вес $/$ рост $\left.^{2}\right)$. 
position in 12 leads with tape speed of $25 \mathrm{~mm} / \mathrm{s}$. ECG analysis was conducted using the Minnesota code (MC) in the 1982-year revised version by two independent experts with participation of a third one in case of disagreement [7].

By educational status the patients were divided in 3 groups: basic, secondary and higher education. Among behavioral habits we evaluated smoking habits (neversmokers, former smokers, current smokers) and alcohol consumption: no alcohol consumption during the last year and excessive alcohol consumption ( $>168 \mathrm{~g}$ of pure ethanol per week for men and $>84 \mathrm{~g}-$ for women). Increased BP ( $\geqslant 140 / 90 \mathrm{~mm} \mathrm{Hg})$ and high $\mathrm{BP}(\geqslant 160 / 95 \mathrm{~mm} \mathrm{Hg})$ were taken into analysis. Heart rate was registered as high at the value of $>80$ b.p.m. TC was considered high at the level of $\geqslant 6,6 \mathrm{mmol} / \mathrm{l}$, which corresponds to the $5^{\text {th }}$ sex-specific quintile of TC distribution in population, and low - at the level under $4,8 \mathrm{mmol} / \mathrm{I}$ ( 1 quintile); increased TG level was registered at the level of $\geqslant 1,7 \mathrm{mmol} /$; low HDL-C - at the values of $\leqslant 1,0 / 1,2 \mathrm{mmol} / \mathrm{l}$ for men and women, respectively.

The following ECG disturbances were considered: major QQS waves - (MC 1-1-1, 1-2-7); and ischemia - major ST-T (MC 4-1, 4-2, 5-1,5-2), without left ventricle hypertrophy (MC 3-1, 3-3); Atrial fibrillation (AF) was registered as the 8-3 MC category. Coronary heart disease (CHD) was detected in cases of major QQS waves, angina pectoris by the Rose questionnaire [8] and major ischemia (ST-T abnormalities).

Mortality was determined using the current mortality register with International Classification of Diseases-8 (ICD). Death cases due to CVD, CHD, any stroke, non-cardiovascular causes and all causes were encoded by the ICD- 8 . A total of $4.7 \%$ persons of the general cohort were lost (duration of follow-up was fixed by the data of drop-out). Altogether 18,085 persons were followed up in the cohort of 35-74 years $(13,046$ men, 5,039 women). To the end of October 2016 the total of 10,650 deaths were registered: 8,726 in men and 1,924 - in women. Table 1 presents absolute numbers of death cases in men and women for 10 and 20 years of follow-up, respectively.

We calculated death rate for the men's cohort for the next 10 years while for the women's cohort - for the next 20 years as the size of the women's cohort was significantly less than that one of men and a number of deaths was limited.

Descriptive statistics (prevalence rates, means and standard errors (SE)) were calculated for main variables; $p<0.05$ values were taken as statistically significant. Hazard ratio (HR) and $95 \%$ confidence intervals $(95 \% \mathrm{Cl})$ were estimated by the multivariate Cox proportional hazards regression for all-cause, CVD, non-
Ожирение классифицировали при ИМТ 230 кг/М². Низкий ИМТ соответствовал уровню меньше $21 \mathrm{kг} / \mathrm{M}^{2}$.

Лабораторные биохимические параметры включали рутинное определение общего холестерина (ХС [ммоль/л]), холестерина липопротеидов высокой плотности (ХС-ЛПВП [ммоль/л]), триглицеридов (ТГ [ммоль/л]). Высокий ХС определялся при уровне $\geqslant 6,6$ ммоль/л, что соответствует 5 полоспецифической квинтили распределения ХС в популяции, низкий - при уровне менее 4,8 ммоль/л (1 квинтиль), повышенный уровень ТГ регистрировался при ТГ $\geqslant 1,7$ ммоль/л, низкий ХС-ЛПВП при значениях $\leqslant 1,0 / 1,2$ ммоль/л для мужчин и женщин, соответственно.

Электрокардиограмма (ЭКГ) регистрировалась в положении лежа на спине в 12 отведениях со скоростью протяжки ленты 25 мм/сек. Анализ ЭКГ проводился с помощью Миннесотского кода (МК) в пересмотре 1982 г. двумя независимыми экспертами с привлечением третьего в случае несогласия [7]. Фибрилляция предсердий (ФП) регистрировалась как категория MК: 8-3. ИБС определялась при наличии больших зубцов QQS, стенокардии по вопроснику Rose [8] и выраженной ишемии миокарда (ST-T).

Смертность определялась согласно действующему регистру смертности: по МКБ-8 кодировались причины смерти от ССЗ, ишемической болезни сердца (ИБС), от любого инсульта, не сердечно-сосудистые причины и все причины. За время наблюдения из когорты было потеряно 4,7\% (время наблюдения фиксировалось датой выбытия) человек. Всего в проспективной когорте 35-74 лет наблюдалось 18085 человек (13046 мужчин и 5039 женщин). На конец октября 2016 г. было зарегистрировано 10650 смертей, 8726 у мужчин и 1924 у женщин. В табл. 1 представлены абсолютные количества случаев смерти у мужчин и женщин за 10 и 20 лет, соответственно. В данном исследовании риск смерти рассчитывался для мужской когорты на ближайшие 10 лет, для женской - на ближайшие 20 лет, поскольку объем женской когорты существенно меньше, чем мужской, и число случаев также ограничено.

Статистический анализ проводился с помощью статистического пакета SAS, версия 6.12. Использовали стандартные методы описательной статистики и известные критерии значимости ( $\chi^{2}$-критерий, t-критерий Стьюдента). Анализ данных смертности проводили в регрессионной модели пропорционального риска (Кокса) отдельно для мужчин и женщин при стандартизации на возраст, город, год скрининга. Данные стратифицированы по образованию.

\section{Результаты}

В табл. 2 представлены характеристики выборок по факторам риска, включенных в исследование, отдельно по полу. Когорты мужчин и женщин не различаются по 
Table 1. Number of death cases due to different causes in men and women for 10 and 20 years Таблица 1. Число случаев смерти от различных причин у мужчин и женщин за 10 и 20 лет

\begin{tabular}{|c|c|c|c|c|c|}
\hline & \multicolumn{5}{|c|}{ Causes of death / Причины смерти } \\
\hline & CHD / ИБС & Stroke / Инсульт & CVD / CC3 & Non-CVD / He CC3 & All causes / Все причины \\
\hline \multicolumn{6}{|l|}{ Men / Мужчины } \\
\hline 10 years / 10 лет & 628 & 234 & 958 & 1050 & 2018 \\
\hline 20 years / 20 лет & 1742 & 733 & 2743 & 2400 & 5316 \\
\hline \multicolumn{6}{|c|}{ Women / Женщины } \\
\hline 10 years / 10 лет & 124 & 81 & 230 & 218 & 457 \\
\hline 20 years / 20 лет & 340 & 236 & 650 & 511 & 1328 \\
\hline
\end{tabular}

Table 2. Prevalence of the risk factors at the baseline

Таблица 2. Распространенность факторов риска на стартовом обследовании

\begin{tabular}{|c|c|c|c|}
\hline Фактор & Men / Мужчины & Women / Женщины & p \\
\hline Mean age, years / Средний возраст, лет (M士SE) & $51.6 \pm 0.02$ & $51.7 \pm 0.02$ & 0.8807 \\
\hline Basic education / Образование ниже среднего, \% & 23.9 & 23.0 & 0.3870 \\
\hline Secondary education / Среднее, \% & 28.1 & 36.8 & 0.0001 \\
\hline Higher education / Высшеe, \% & 48.0 & 40.2 & 0.0001 \\
\hline Never-smokers / Никогда не курили, \% & 23.2 & 81.2 & 0.0001 \\
\hline Former smokers / Бросили курить, \% & 28.8 & 7.0 & 0.0001 \\
\hline Current smokers / Курят сейчас, \% & 42.3 & 9.8 & 0.0001 \\
\hline No alcohol consumption for 1 year / Не употребляли алкоголь в течение года, \% & 12.0 & 29.2 & 0.0001 \\
\hline Excessive alcohol consumption / Употребляли чрезмерно, \% & 12.5 & 2.7 & 0.0001 \\
\hline Heart rate > 80 bpm / ЧСС >80 уд/мин, \% & 9.8 & 12.4 & 0.0005 \\
\hline High ВР ( $\geqslant 160 / 95$ mm Hg) / Высокое АД ( $\geqslant 160 / 95$ мм рт.ст.), \% & 32.5 & 35.4 & 0.0064 \\
\hline Increased BP ( $\geqslant 140 / 90$ mm Hg) / Повышенное АД ( $\geqslant 140 / 90$ мм рт.ст.), \% & 55.2 & 55.4 & 0.8609 \\
\hline СНD / ИБС, \% & 17.5 & 19.9 & 0.0007 \\
\hline $\mathrm{AF} / \Phi \Pi, \%$ & 1.62 & 1.31 & 0.0979 \\
\hline High TC ( $\geqslant 6.6 \mathrm{mmol} / \mathrm{L})$ / Высокий XС ( $\geqslant 6,6$ ммоль/л), \% & 17.8 & 24.4 & 0.0001 \\
\hline Low TC ( $\leqslant 4.8$ mmol/L) / НизкийXC (\$4,8 ммоль/л), \% & 22.0 & 17.7 & 0.0001 \\
\hline 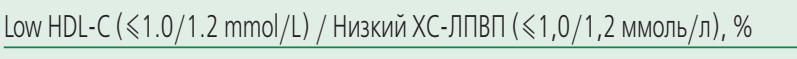 & 17.8 & 23.7 & 0.0001 \\
\hline High TG ( $\geqslant 1.7$ mmol/L) / Высокие ТГ ( $\geqslant 1,7$ ммоль/л), \% & 21.8 & 18.9 & 0.0023 \\
\hline High BMI $\left(\geqslant 30.0 \mathrm{~kg} / \mathrm{m}^{2}\right) / 0$ жирение $\left(\right.$ ИМТ $\geqslant 30,0$ кг $\left./ \mathrm{M}^{2}\right), \%$ & 14.6 & 35.9 & 0.0001 \\
\hline Low BMI $\left(\leqslant 23 \mathrm{~kg} / \mathrm{m}^{2}\right)$ / Низкий ИМт $\left(\leqslant 23,0\right.$ кг $\left./ \mathrm{M}^{2}\right)$, \% & 20.2 & 12.3 & 0.0001 \\
\hline
\end{tabular}

CVD, stroke and CHD mortality separately for men and women. The model included age, survey period, city, education, alcohol consumption, smoking status, high and increased BP, lipids profile, obesity and abdominal obesity, fasting glucose, CHD. Data were analyzed with SAS version 6.12 возрасту, частоте повышенного АД. Отмечается сильная диссоциация по уровню образования. Количество мужчин и женщин с образованием ниже среднего практически одинаково, однако среди мужчин было больше лиц с высшим образованием, а среди женщин - со средним, различия статистически значимы. Как и ожидалось, на- 


\section{Results}

Initial characteristics of the subjects (men and women, separately) are presented in Table 2 . The cohorts of men and women were not differed by age and prevalence of increased BP. The strong dissociation by educational status was observed. A number of basiceducated men and women was nearly identical, however men more often had higher education while women - secondary one, differences were statistically significant. Behavioral risk factors (smoking and alcohol consumption) significantly prevailed in the men's cohort. On the contrary, the women's cohort revealed higher prevalence of increased total cholesterol, reduced HDL-C levels and obesity. Every fifth person in the men's and $12 \%$ in the women's cohorts had low body weight. High heart rate was more often detected in women.

Analysis of age-adjusted dynamics of mortality rates by 1,000 person-years of follow-up (Table 3 ) had demonstrated significantly higher rates of mortality due to CHD, CVD, other non-CVD causes and all causes in men, while women only died of stroke more often than men. Mortality from CHD in persons aged 35-64 years were significantly higher in men and after 65 years of age - insignificantly higher in women. Gender differences in stroke-related mortality were insignificantly in age 35-64. But women above 65 years died from stroke twice as frequently as men, this was statistically significant: absolute risk (AR) $12.35(95 \% \mathrm{Cl}$ 9.68-15.02) and AR 6.29 (95\%Cl 3.11-9.47), respectively. We have found no significant gender differences in cardiovascular mortality, however women of блюдаются существенные различия в поведенческих факторах риска с явным преобладанием последних в мужской когорте (курение и чрезмерное потребление алкоголя). Напротив, высокий холестерин, низкий ХС-ЛПВП и, особенно, ожирение чаще регистрировались в женской когорте. Следует отметить, что каждый пятый в мужской когорте и 12\% в женской имели низкий вес. Высокая ЧСС чаще выявлялась у женщин.

Анализ возрастной динамики рисков смерти на 1000 человеко-лет наблюдения (ЧЛН) (табл. 3) показал, что От ИБС, ССЗ, других не ССЗ причин и от всех причин достоверно чаще умирали мужчины, и лишь от инсульта женщины. Вместе с тем, смертность от ИБС статистически значимо чаще отмечалась у мужчин в возрасте 35-64 года, и только после 65 лет начинала преобладать у женщин, хотя и не достигая статистической значимости. Смертность от инсульта, напротив, в 35-54 года была несколько выше у мужчин, а в группе 55-64 уже у женщин, но после 65 лет женщины умирали от инсульта в 2 раза чаще мужчин, что статистически значимо: абсолютный риск (АР) 12,35; 95\% доверительный интервал (ДИ) 9,68-15,02 против АР 6,29 (95\%ДИ 3,11-9,47), соответственно. Статистически значимых гендерных различий в смертности от ССЗ отмечено не было. От других не ССЗ причин чаще умирали мужчины, так же как и от всех причин. Вообще, несмотря на то, что риск смерти в женской когорте оценивался в промежуток времени, в два раза превышающий таковой у мужчин, преобладание смертности в мужской когорте очевидно.

Результаты анализа ассоциаций (табл. 4-8), показали, что, во-первых, при разных причинах действует некоторое число одних и тех же факторов, остальные могут ме-

Table 3. Mortality rate per 1000 person-year by sex and age groups in 10 years (males), 20 years (females), AR $(95 \% \mathrm{Cl})$

Таблица 3. Риск умереть от различных причин для мужчин (10 лет) и женщин (20 лет) на 1000 человеко-лет наблюдений в зависимости от возраста, АР (95\%ди)

\begin{tabular}{|c|c|c|c|c|c|}
\hline $\begin{array}{l}\text { Age groups (years) / } \\
\text { Возрастные группы (лет) }\end{array}$ & СНD / ИБС & Stroke / Инсульт & CVD / CC3 & Non CVD / He CC3 & All causes / Bсе причины \\
\hline \multicolumn{6}{|c|}{ Males, 10 years of follow-up / Мужчины, 10 лет наблюдения } \\
\hline $35-44$ & $1.94(1.49-2.39)$ & $0.60(0.35-0.85)$ & $2.84(2.29-3.39)$ & $5.05(4.32-5.78)$ & $7.95(7.03-8.87)$ \\
\hline $45-54$ & $4.82(4.25-5.39)$ & $1.95(1.60-2.30)$ & $7.50(6.79-8.21)$ & $8.43(7.69-9.17)$ & $16.03(14.99-17.07)$ \\
\hline $55-64$ & $9.63(8.41-10.85)$ & $3.44(2.71-4.17)$ & $14.32(12.83-15.81)$ & $13.39(11.94-14.84)$ & $27.71(25.63-29.79)$ \\
\hline $65-74$ & $18.02(12.63-23.41)$ & $6.29(3.11-9.47)$ & $27.66(21.00-34.32)$ & $21.37(15.51-27.23)$ & $51.55(42.44-60.66)$ \\
\hline $35-74$ & $4.73(4.36-5.10)$ & $1.76(1.54-1.98)$ & $7.19(6.74-7.64)$ & $8.06(7.57-8.55)$ & $15.36(14.69-16.03)$ \\
\hline \multicolumn{6}{|c|}{ Females, 20 years of follow-up / Женщины, 20 лет наблюдения } \\
\hline $35-44$ & $0.62(0.33-0.91)$ & $0.24(0.06-0.42)$ & $0.93(0.58-1.28)$ & $2.53(1.94-3.12)$ & $3.91(3.18-4.64)$ \\
\hline $45-54$ & $1.44(0.99-1.89)$ & $1.83(1.32-2.34)$ & $3.81(3.08-4.54)$ & $4.49(3.71-5.27)$ & $9.52(8.36-10.68)$ \\
\hline $55-64$ & $6.84(5.80-7.88)$ & $4.00(3.20-4.80)$ & $12.34(10.93-13.75)$ & $8.30(7.14-9.46)$ & $23.19(21.27-25.11)$ \\
\hline $65-74$ & $17.78(14.57-20.99)$ & $12.35(9.68-15.02)$ & $33.30(28.91-37.69)$ & $17.18(14.02-20.34)$ & $59.36(53.50-65.22)$ \\
\hline $35-74$ & $3.23(2.90-3.56)$ & $2.28(1.99-2.57)$ & $6.24(5.77-6.71)$ & $5.08(4.65-5.51)$ & $12.99(12.30-13.68)$ \\
\hline \multicolumn{6}{|c|}{ AR - absolute risk, CI - confidence interval, CHD - coronary heart disease, CVD - cardiovascular diseases } \\
\hline \multicolumn{6}{|c|}{ АР - абсолютный риск, ДИ - доверительный интервал, ИБС - ишемическая болезнь сердца, ССЗ - сердечно-сосудистые заболевания } \\
\hline
\end{tabular}


the eldest age group died more often: HR 33.30 (95\% Cl 28.91-37.69) vs HR 26.66 (95\%Cl 21.0034.32). All-cause mortality and mortality from nonCVD causes were higher in men. In general, in spite of death risk in the women's cohort was estimated for a time period twice as long as in the men, higher mortality rates in the men's cohort was obvious.

It is observed that different causes of death associated with different number of the same factors while other predictors could be different (Table 4-8). It also found that mortality in men and women was associated with different factors and with different strength. няться. Во-вторых, у мужчин и женщин могут ассоциироваться со смертностью разные факторы и с разной силой.

Стандартизованные по возрасту, городу, когорте наблюдения и основным факторам риска виды смертности представлены для мужчин и женщин в табл. 4 (смертность от ИБС), табл. 5 (смертность от инсульта), в табл. $6,7,8$ (смертность от не СС3, от СС3 и смертность от всех причин, соответственно).

Статистически значимых факторов, определяющих смертность от ИБС среди мужской когорты, осталось девять (табл. 4), среди которых по показателю отношения

Table 4. Associations of basic risk factors and CHD-related mortality rates in Russian men and women adjusted for age, city of residence, time of screening, stratified by educational status, HR $(95 \% \mathrm{Cl})$

Таблица 4. Стандартизованные по возрасту, городу и периоду скрининга, стратифицированные по образованию ассоциации основных факторов риска и смертности от ИБС у мужчин и женщин, ОР (95\%ДИ)

\begin{tabular}{|c|c|c|c|c|}
\hline Parameter / Параметр & Males / Мужчины & $\mathrm{p}$ & Females / Женщины & p \\
\hline Quit smoking / Бросили курить & $1.548(1.179-2.033)$ & 0.0017 & $1.582(1.073-2.333)$ & 0.0206 \\
\hline Smoking / Курят & $2.247(1.747-2.892)$ & 0.0001 & $2.048(1.435-2.922)$ & 0.0001 \\
\hline Without alcohol consumption / Не употребляли алкоголь & & & $1.290(1.023-1.627)$ & 0.0315 \\
\hline Excessive alcohol consumption / Употребляли чрезмерно & & & $2.826(1.034-7.727)$ & 0.0429 \\
\hline High heart rate / Повышенная ЧСС & $1.282(1.044-1.574)$ & 0.0177 & & \\
\hline High ВР / Высокое АД & $1.781(1.430-2.218)$ & 0.0001 & $2.039(1.621-2.565)$ & 0.0001 \\
\hline CHD / ИБС & $3.227(2.711-3.841)$ & 0.0001 & $1.627(1.293-2.048)$ & 0.0001 \\
\hline AF / ФП & $1.666(0.921-3.013)$ & 0.0915 & $3.616(1.888-6.927)$ & 0.0001 \\
\hline High TC / Высокий XС & $1.428(1.188-1.717)$ & 0.0001 & $1.419(1.136-1.773)$ & 0.0020 \\
\hline Low TC / НизкийXC & $1.265(1.035-1.545)$ & 0.0216 & $1.348(1.070-1.697)$ & 0.0112 \\
\hline Low BMI / Низкий ИМТ & $1.250(1.015-1.538)$ & 0.0353 & & \\
\hline
\end{tabular}

Table 5. Stroke mortality among Russian males and females standardized by age, city, screening and main risk factors stratified by educational, HR $(95 \% \mathrm{Cl})$

Таблица 5. Стандартизованные по возрасту, городу и периоду скрининга, стратифицированные по образованию ассоциации основных факторов риска и смертности от инсульта у мужчин и женщин, ОР (95\%ДИ)

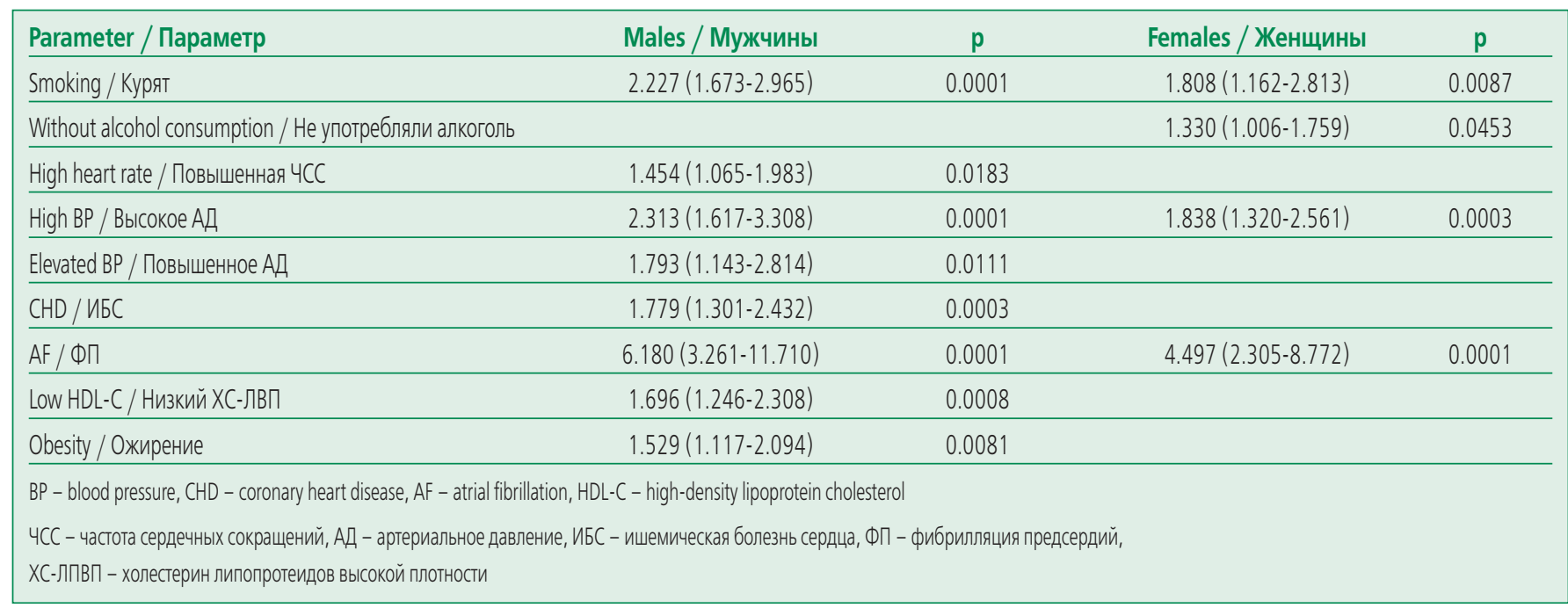


Table 6. CVD mortality among Russian males and females standardized by age, city, screening and main risk factors stratified by educational, $\mathrm{HR}(95 \% \mathrm{Cl})$

Таблица 6. Стандартизованные по возрасту, городу и периоду скрининга, стратифицированные по образованию ассоциации основных факторов риска и смертности от ССЗ у мужчин и женщин, ОР (95\%дИ)

\begin{tabular}{|c|c|c|c|c|}
\hline Parameter / Параметр & Males / Мужчины & $p$ & Females / Женщины & $p$ \\
\hline Quit smoking / Бросили курить & $1.274(1.029-1.576)$ & 0.0261 & $1.521(1.148-2.015)$ & 0.0035 \\
\hline Smoking / Курят & $1.974(1.626-2.396)$ & 0.0001 & $1.911(1.467-2.489)$ & 0.0001 \\
\hline Without alcohol consumption / Не употребляли алкоголь & $1.263(1.019-1.565)$ & 0.0332 & $1.355(1.146-1.603)$ & 0.0004 \\
\hline High heart rate / $4 \mathrm{CC}$ & $1.311(1.114-1.543)$ & 0.0011 & $1.200(0.984-1.463)$ & 0.0717 \\
\hline High ВР / Высокое АД & $1.982(1.655-2.374)$ & 0.0001 & $2.071(1.755-2.445)$ & 0.0001 \\
\hline Elevated ВР / Повышенное АД & $1.222(0.997-1.496)$ & 0.0533 & & \\
\hline СНD / ИБС & $2.753(2.384-3.179)$ & 0.0001 & $1.545(1.307-1.825)$ & 0.0001 \\
\hline$\underline{\mathrm{AF}} /$ ФП & $3.409(2.360-4.923)$ & 0.0001 & $4.708(3.134-7.071)$ & 0.0001 \\
\hline High TC / ВысокийXС & $1.400(1.204-1.628)$ & 0.0001 & $1.188(1.007-1.402)$ & 0.0411 \\
\hline Low HDL-C / Низкий ХС-ЛВП & $1.344(1.144-1.579)$ & 0.0003 & & \\
\hline Obesity / Ожирение & $1.146(0.963-1.363)$ & 0.1247 & & \\
\hline Low BMI / Низкий ИМТ & $1.281(1.079-1.522)$ & 0.0046 & & \\
\hline \multicolumn{5}{|c|}{ BP - blood pressure, CHD - coronary heart disease, AF - atrial fibrillation, TC - total cholesterol, HDL-C - high-density lipoprotein cholesterol, BMI - body mass index } \\
\hline \multicolumn{5}{|c|}{$\begin{array}{l}\text { чСС - частота сердечных сокращений, АД - артериальное давление, ИБС - ишемическая болезнь сердца, ФП - фибрилляция предсердий, } \\
\text { ХС-лПВП - холестерин липопротеидов высокой плотности, ИМТ - индекс массы тела }\end{array}$} \\
\hline
\end{tabular}

Table 7. Non-CVD mortality among Russian males and females standardized by age, city, screening and main risk factors stratified by educational, $\mathrm{HR}(95 \% \mathrm{Cl})$

Таблица 7. Стандартизованные по возрасту, городу и периоду скрининга, стратифицированные по образованию ассоциации основных факторов риска и смертности от не ССЗ у мужчин и женщин, ОР (95\%ДИ)

\begin{tabular}{|c|c|c|c|c|}
\hline Parameter / Параметр & Males / Мужчины & $\mathrm{p}$ & Females / Женщины & $\mathrm{p}$ \\
\hline Smoking / Курят & $1.613(1.348-1.930)$ & 0.0001 & $1.382(1.041-1.835)$ & 0.0251 \\
\hline Without alcohol consumption / Не употребляли алкоголь & & & $1.230(1.010-1.497)$ & 0.0395 \\
\hline Excessive alcohol consumption / Употребляли алкоголь чрезмерно & $1.202(1.001-1.445)$ & 0.0493 & & \\
\hline High heart rate / Повышенная ЧСС & $1.379(1.177-1.616)$ & 0.0001 & $1.245(0.993-1.562)$ & 0.0577 \\
\hline High ВР / Высокое АД & & & $1.285(1.065-1.550)$ & 0.0089 \\
\hline Elevated ВР / Повышенное АД & $1.186(1.040-1.353)$ & 0.0110 & & \\
\hline Low TC / НизкийXC & $1.216(1.045-1.415)$ & 0.0113 & & \\
\hline Low BMI / Низкий ИМТ & $1.678(1.460-1.930)$ & 0.0001 & & \\
\hline
\end{tabular}

Causes of death in men and women adjusted for age, the city of residence, cohort of follow-up and principal risk factors are reported in Table 4 (mortality from CHD), Table 5 (stroke-related mortality), Tables 6,7,8 (mortality from CVD, non-CVD diseases and allcause mortality, respectively).

We had detected 9 statistically significant factors influencing CHD-related death in the men's cohort (Table 4), the most significant were the current smoking ( $\mathrm{HR}$ $2.25 ; 95 \% \mathrm{Cl} 1.75-2.89 ; \mathrm{p}=0.0001)$ and CHD presence at the start of the study (HR 3.23; 2.71-3.84; $\mathrm{p}=0.0001$ ). In other words, CHD-diagnosed men have рисков (ОР) наиболее значительны курение в настоящее время ОР 2,25; 95\%ДИ 1,75-2,89; $p=0,0001$ и наличие ИБС на старте исследования (ОР 3,23; 95\%ДИ 2,71-3,84; $=0,0001)$, иначе говоря, вероятность умереть от ИБС у мужчин с диагнозом ИБС в ближайшие 10 лет в 3 раза выше, а среди курящих - в 2,2 раза выше по сравнению с мужчинами, не имеющими данного фактора. Среди других показателей в мужской когорте следует обратить внимание на высокое АД (ОР 1,78; $95 \%$ ДИ 1,43-2,22; $p=0,0001$ ) и высокий холестерин (ОР 1,43; 95\%ДИ 1,19-1,72; $p=0,0001)$. Женская когорта характеризуется несколько другим набором значимых пере- 
Table 8. All-cause mortality among Russian males and females standardized by age, city, screening and main risk factors stratified by educational, HR $(95 \% \mathrm{Cl})$

Таблица 8. Стандартизованные по возрасту, городу и периоду скрининга, стратифицированные по образованию ассоциации основных факторов риска и смертности от всех причин у мужчин и женщин, ОР (95\%ДИ)

\begin{tabular}{|c|c|c|c|c|}
\hline Parameter / Параметр & Males / Мужчины & $p$ & Females / Женщины & p \\
\hline Quit smoking / Бросили курить & $1.217(1.050-1.411)$ & 0.0090 & $1.233(0.998-1.524)$ & 0.0521 \\
\hline Smoking / Курят & $1.799(1.577-2.051)$ & 0.0001 & $1.674(1.399-2.003)$ & 0.0001 \\
\hline Without alcohol consumption / Не употребляли алкоголь & $1.216(1.038-1.423)$ & 0.0153 & $1.277(1.132-1.441)$ & 0.0001 \\
\hline \multicolumn{2}{|c|}{ Excessive alcohol consumption / Употребляли алкоголь чрезмерно } & & $1.605(0.942-2.737)$ & 0.0820 \\
\hline High heart rate / Повышенная ЧСС & $1.332(1.189-1.492)$ & 0.0001 & $1.210(1.049-1.395)$ & 0.0088 \\
\hline High ВР / Высокое АД & $1.440(1.277-1.623)$ & 0.0001 & $1.656(1.477-1.857)$ & 0.0001 \\
\hline Elevated ВР / Повышенное АД & $1.169(1.029-1.328)$ & 0.0161 & & \\
\hline CHD / ИБС & $1.882(1.679-2.109)$ & 0.0001 & $1.372(1.211-1.554)$ & 0.0001 \\
\hline AF / ФП & $2.373(1.725-3.264)$ & 0.0001 & $3.194(2.247-4.538)$ & 0.0001 \\
\hline High TG / Высокие ТГ & $1.256(1.122-1.407)$ & 0.0001 & & \\
\hline Low TG / Низкие TГ & $1.158(1.029-1.303)$ & 0.0153 & & \\
\hline Low HDL-C / Низкий ХС-ЛВП & $1.146(1.018-1.290)$ & 0.0236 & $1.150(1.015-1.301)$ & 0.0278 \\
\hline Low BMI / Низкий ИМТ & $1.526(1.368-1.702)$ & 0.0001 & $1.211(0.990-1.482)$ & 0.0620 \\
\hline \multicolumn{5}{|c|}{ BP - blood pressure, CHD - coronary heart disease, AF - atrial fibrillation, TC - total cholesterol, TG - triglycerides, HDL-C - high-density lipoprotein cholesterol, BMI - body mass index } \\
\hline \multicolumn{5}{|c|}{ чСС - частота сердечных сокращений, АД - артериальное давление, ИБС - ишемическая болезнь сердца, ФП - фибрилляция предсердий, ТГ - триглицериды, } \\
\hline
\end{tabular}

3-time and current smokers - 2.2-time higher possibility of death in the next 10 years as compared to men without these risk factors. As for the other risk parameters in the men's cohort high BP $(\mathrm{HR} 1.78 ; 95 \% \mathrm{Cl}$ 1.43-2.22; $p=0.0001)$ and high TC level (HR 1.43; $1.19-1.72 ; p=0.0001$ ) are noticed. The women's cohort was characterized by a somewhat other pattern of significant variables, however such factors as smoking (HR 2.05; 1.43-2.92; $p=0.0001$ ), high BP (HR 2.04; $1.62-2.56 ; p=0.0001)$ and history of CHD were also associated with risk of CHD-related death in 20 years. At the same time it should be noted that the most critical factor contributing CHD-related mortality in women was AF (HR 3.62; 1.89-6.93; $p=0.0001$ ). Excessive alcohol consumption must also be mentioned in women. Abstinents of both genders were also demonstrated to have higher mortality rates as compared to persons with little and moderate alcohol consumption.

Factors associated with high risk of stroke-related death (Table 5) in men were conventional: AF (HR $6.18 ; 95 \% \mathrm{Cl} 3.26-11.71 ; p=0.0001)$, smoking, high BP, obesity, low HDL-C and CHD. Only 3 of these 5 parameters statistically significantly associated with death from stroke in women and $\mathrm{AF}$ was also the first in line ( $\mathrm{HR} 4.50 ; 95 \% \mathrm{Cl} 2.30-8.77 ; p=0.0001)$. Obesity and lipids profile had not selected.

Mortality from CVD (Table 6) summarizes cardiovascular causes including peripheral artery diseases, valvular diseases and so on. A number of highly signif- менных, однако курение (ОР 2,05; 95\%ДИ 1,43-2,92; $p=0,0001)$, высокое АД (ОР 2,04; 95\%ДИ 1,62-2,56; $\mathrm{p}=0,0001)$, наличие ИБС в анамнезе, последнее хотя в 2 раза менее выраженное, чем у мужчин, но статистически высоко значимое, также относятся к показателям риска смерти от ИБС в течение 20 лет. Наиболее существенным фактором, формирующим смертность от ИБС у женщин, является ФП (ОР 3,62, 95\%ДИ 1,89-6,93; $\mathrm{p}=0,0001)$, и нельзя не отметить чрезмерное потребление алкоголя. Вместе с тем, абстиненты обоего пола также имеют тенденцию умереть быстрее по сравнению с мало и умеренно пьющими, но у женщин этот показатель значим на уровне $5 \%$.

Характеристика факторов, ассоциированных с высоким риском смертности от инсульта (табл. 5) у мужчин, включает все общепринятые ФР: ФП (ОР 6,18; 95\%ДИ 3,26-11,71; $=0,0001)$, курение, высокое АД, ожирение, низкий ХС-ЛПВП и наличие ИБС. У женщин только три из 5 достоверных показателей статистически значимо ассоциируются с этой причиной, и первое место также занимает ФП (ОР 4,50, 95\%ДИ 2,30-8,77; $p=0,0001$ ). Ожирение и липидные показатели не вошли в модель.

Смертность от СС3 (табл. 6) является интегральным показателем, обобщающим сердечно-сосудистые причины, включая заболевания крупных сосудов, клапанную патологию и т.д. В мужской и женской когортах основные статистически значимые ассоциации с ФР совпадают, но у мужчин добавляются показатели липидного профиля, тогда как у женщин наблюдается лишь незначительное 
icant associations coincided in the men's and women's cohorts, at that lipid profile variables were only significant in men while in the women's cohort the high TC level was of borderline significance. High heart rate and low body weight in men must be underlined as unfavorable predictors which increased CVD mortality rates.

In case of non-CVD deaths smoking was the only common factor statistically significantly associated with this mortality in men and women (Table 7). It should be noted that smoking was more frequently associated with risk factors in the men's cohort where highly significant associations between high heart rate, low body weight and smoking had been reported, low TC and excessive alcohol consumption were also significant. In women cardiovascular parameters such as high BP and high heart rate and also abstinence were more significant.

Almost all the factors included in the analysis contributed to total mortality (Table 8 ) among men and women with exclusion for obesity and excessive alcohol consumption in men (which did not achieve the threshold of significance) and excessive alcohol consumption, high cholesterol and obesity - in women, the last two parameters were not even selected.

\section{Discussion}

Analysis of death rates per 1,000 person-years of follow-up had demonstrated atherosclerosis-related mortality to be more typical of the men's cohort than of the women's one with disappearance of statistically significance after 65 years of age only. However a similar trend was reported for non-CVD deaths which can be explained by higher rates of mortality from external causes in younger age in the men's population possibly due to smoking and excessive alcohol consumption. Analysis of associations of death risks with the causes had demonstrated their dependence on age. In other words, with increasing age the gender difference decrease. This is apparently associated with premature CVD death and external causes in the young men's population. Stroke-related mortality in the women's cohort also had age features. So, death rates after 65 years were statistically significant more prevailed in the women's cohort which can be explained by higher BP level in women of this age.

Higher mortality rates in men as compared to women have been known [9]. Our study was not aimed at questions of gender differences. Although this subject was presented and discussed in many reviews in particular of M. Luy, Y. Mingava and V. Zarulli et al. (2018) [10,11].

However the fact of less number of risk factors associated with death in the women's cohort deserves влияние высокого XС на смертность от ССЗ. Следует подчеркнуть, что высокая ЧСС и низкая масса тела у мужчин являются неблагоприятными признаками, повышающими риск смертности от ССЗ.

Общим признаком, статистически значимо связанным с не сердечно-сосудистой смертностью (табл. 7) для мужчин и женщин является только курение. Следует заметить, что вообще этот вид смертности более связан с факторами риска у мужчин, в когорте которых отмечены высоко достоверные ассоциации с высокой ЧСС, низким ИМТ, курением, где значимы также низкий ХС и чрезмерное потребление алкоголя. Для женщин более характерны ассоциации с высоким АД, ЧСС и абстиненцией.

Практически все факторы, включенные в анализ, формируют общую смертность (табл. 8) у мужчин и у женщин. Исключение - у мужчин ожирение и чрезмерное потребление алкоголя, не достигшие значимости; у женщин - чрезмерное потребление алкоголя, высокий холестерин и ожирение, последние два параметра вообще не были отобраны в модель.

\section{Обсуждение}

Более высокая смертность среди мужчин по сравнению с женщинами давно известна, и как указывали J. Lorber и L.J. Moore, еще в 20-х годах прошлого века «Women get sicker, but men die quicker», что означает: «женщины болеют, но мужчины умирают быстрее» [9]. Вопросы гендерных различий хорошо представлены в работах M. Luy и Y. Mingava и V. Zarulli с соавт. $[10,11]$.

Анализ возрастных трендов смертности на 1000 человеко-лет наблюдений показал, что причины смерти, связанные с атеросклерозом, более характерны для мужской когорты, чем для женской, и только после 65 лет статистическая значимость различий исчезает.

В нашем исследовании был проведен анализ 17 переменных - потенциальных факторов риска - с целью определить их прогностическое значение для различных причин смерти. Было обнаружено, что мужчины имеют большее количество ФР, которые ассоциируются с любой причиной смертности. Большее число факторов, влияющих на смертность, на наш взгляд, характеризует, большую распространенность факторов риска среди мужской когорты. В то же время есть факторы, общие для всех причин, и есть менее значимые, но имеющие специфическое сродство только с определенными причинами. Так, для смертности от всех причин наиболее значимыми и совпадающими для обоих полов оказались курение, высокое АД, ИБС в анамнезе и наличие ФП. При этом связь между курением, ИБС и смертностью была более выражена в мужской когорте, а высокое АД и ФП более характерны для женской когорты. Таким образом, даже единые факторы для обоих полов с разной силой ассоциируются со смертностью. Если принять во внимание, 
attention in line with other explanations of the gap in mortality rates between the genders. We have analyzed 17 variables - potential risk factors to estimate their prognostic value for cause-specific death. Analysis was conducted in both genders separately because of significant gradient in mortality rates between men and women are typical of our population. Men were found to have a large number of risk factors which were associated with any cause of death. A larger number of risk factors contributing to mortality in the men's population can partly explain to our opinion higher mortality rate.

The most important for men's cohort were RF associated with most cause-specific deaths: smoking, high heart rate, high $B P, C H D, A F$, high $T C$ and low HDL whereas for women's one - only smoking, high BP, $\mathrm{CHD}$, and AF. But the strength of associations is more pronounced in men's cohort. Uncertainty of associations of cholesterol levels with mortality rates had also been considered by researchers, in our work this factor was not even selected in the models in the women's cohort. Indirect correlation between low TC level and mortality rate in Russia had been reported earlier. J. Cai et al. showed association of cholesterol level and allcause mortality was J-shaped and statistically significant for all men [12]. In women correlation was nonlinear and statistically insignificant, but became statistically significant at other risk factors correcting. Korean researchers similarly analyzed correlation of TC level with CVD mortality, and had found U-shape associations after adjustment for sex, age, smoking status, alcohol consumption, BMI, BP, triglycerides and HDL-C. The authors concluded that statins for primary prevention must be prescribed with cautiousness in adult Koreans at low cardiovascular risk [13].

High heart rate and low BMI which demonstrated significant associations with mortality rates in men while in women correlation with high heart rate was less significant and associations with low BMI did not achieve statistical significance. The received data demonstrate not only the TC level but also BMI to be $U$-shape associated with all-cause mortality rate. This fact was the subject of wide discussion in scientific community. A. Romero-Corral et al. published systematic review of studies evaluated associations between obesity and all-cause mortality rates. Authors had analyzed 40 trials which included a total of 250,152 patients with mean follow-up of 3.8 years. Patients with low BMI $\left(<20 \mathrm{~kg} / \mathrm{m}^{2}\right)$ were at increased risk for allcause mortality and CVD-related death [14]. The authors supposed that current recommendations to keep $\mathrm{BMI}$ in a lower half of the target range are only acceptable for a definitely healthy part of a population [15]. Earlier described Russian data confirmed the view of a number of researchers, in particular, C. Flegal et что у мужчин воздействие повышенного АД начинается со 140/90 мм рт.ст., а у женщин - со 160/95 мм рт.ст., то этот факт может служить дополнительным аргументом в пользу более раннего повреждающего действия ФР на мужской организм. Неоднозначность ассоциаций различных уровней XС со смертностью в России была описана ранее. Как показали J. Саі с соавт. [12], ассоциации холестерина и общей смертности J-образны и статистически значимы для всех мужчин. В специфических для страны отношениях холестерин был статистически значимо, линейно и положительно связан с общей смертностью у мужчин из России и США. Для женщин соотношение было нелинейным, но статистически не значимым. Для польских женщин существовала статистически значимая обратная связь. Смертность от ИБС и смертность от ССЗ линейно увеличивались с увеличением уровня холестерина у мужчин из Польши, России и США и общей совокупности мужчин, но у женщин не было выявлено ассоциаций вообще.

Аналогичная U-образная зависимость между XC и смертностью от ССЗ была продемонстрирована корейскими исследователями после стандартизации на другие ФР. Основываясь на результатах этого исследования, авторы предупреждают, что следует проявлять осторожность при назначении статинов для первичной профилактики среди людей с низким сердечно-сосудистым риском у взрослых корейцев [13].

Высокая ЧСС и низкий ИМТ сильнее связаны со смертностью у мужчин по сравнению с женщинами. Ранее было показано, что ИМТ U-образно ассоциируются со смертностью от всех причин. Так, A. Romero-Corral с соавт. (2006 г.) анализировали 40 исследований с 250152 пациентами, со средним периодом наблюдения 3,8 года. Пациенты с низким ИМТ $<20$ кг/M² имели повышенный риск общей смертности и смертности от ССЗ [14]. В крупном проспективном исследовании было выявлено, что смертность от респираторных заболеваний, травм и от старости была сосредоточена в категории ИМТ меньше 18,5. Тем не менее, авторы полагают, что текущие рекомендации по поддержанию ИМТ в нижней части желаемого диапазона остаются приемлемыми для безусловно здорового населения [15]. Российские данные, описанные ранее, подтверждают точку зрения, высказанную многими исследователями, в частности, в 2010 г. C. Flegal с соавт. о том, что наименьшие ассоциации между ИМТ

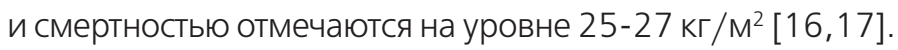

Ассоциации факторов риска и смертности от ССЗ во многом совпадают с ассоциациями, полученными при анализе смертности от всех причин, поскольку именно ССЗ в России составляет половину в структуре общей смертности [18]. Общие для обоих полов факторы: курение, высокое АД, ИБС в анамнезе и ФП. Для мужчин важно также влияние высокого ХС и низкого ХС-ЛПВП, что характерно для болезней, связанных с атеросклерозом. 
al. that the smallest associations between BMI and mortality are noted at the level of $25-27 \mathrm{~kg} / \mathrm{m}^{2}$ $[16,17]$.

Associations of CVD-related death with risk factors are to a large extent coincide with associations revealed at all-cause mortality assessment, as just CVD comprise more than half of total mortality in Russia [18]. The general factors for both genders were the follows: smoking, high BP, history of CHD and AF presence. High TC and low HDL-C levels were also significant in men being typical of atherosclerosis-related diseases, low body weight in men was a significant contributor as well.

There were three RF general for men and women in case of CHD-related deaths: smoking, high BP and history of CHD. However, thin men only died from CHD. It is interesting that AF was significantly associated with CHD-related mortality in women at that it had not even achieved the level of statistical significance in men. Similarly women demonstrated association with excessive alcohol consumption at the significance value of $5 \%$ while men did not. Whether this fact was a result of high susceptibility of the female organism to alcohol is a matter for further investigations.

Stroke is the leading cause of acquired severe disability in adults worldwide and the second cause of death in patients after 60 years Eight factors in the men's and five in the women's cohorts influenced stroke-related mortality in our study. High statistical significance was equal for 3 factors: smoking, AF and high BP. Another 5 factors associated with stroke-related death in the men's cohort: history of CHD, low $H D L-C$, increased $B P$, obesity and high heart rate and another two - in the women's cohort: history of CHD and abstinence during the previous year. Our data are consistent with other studies results. The triad of factors such as smoking, AF and high BP have rather wide evidentiary basis and were included in majority of guidelines for stroke management [19]. Obesity being an independent predictor of ischemic stroke, especially in younger patients, is considered as one of the basic risk factors for stroke.

Our previous works did not in detail present the question of non-CVD-related mortality but taking into account common risk factors we analyzed this cause along with the others. Only smoking was the common factor for men and women. The most significant factor in men was low BMI, then smoking and high heart rate. Low HDL-C and increased (but not high) BP were also significant. Excessive alcohol consumption was of borderline significance. The pattern of non-CVD mortality in women also comprised high BP as a risk factor. Taking into account that this cause of death contains cancer of different localizations and external causes the received pattern of RF associated with the cause is quite natural:
Для смертности от ИБС общими ФР для мужчин и женщин были три параметра: курение, высокое АД и ИБС в анамнезе. Интересно, что ФП у женщин имеет высоко значимые ассоциации, а у мужчин даже не достигает степени статистической значимости. Аналогично в женской когорте на уровне 5\% значимости выявлены ассоциации с чрезмерным употреблением алкоголя, чего не найдено в мужской когорте. Является ли этот факт следствием большей уязвимости женщин для алкоголя? Ответ на этот вопрос требует дополнительного рассмотрения.

Инсульт является ведущей причиной приобретенной тяжелой инвалидности у взрослых во всем мире, и второй причиной смерти у пациентов старше 60 лет. Всемирная организация здравоохранения называет инсульт причиной следующей эпидемии XXI века. В нашем исследовании смертность от инсульта формируют 8 факторов в мужской когорте и 5 - в женской. Высокая статистическая значимость ассоциаций одинакова для курения, ФП и высокого АД. У мужчин важно наличие ИБС в анамнезе, низкий ХС-ЛПВП, повышенное АД, ожирение и высокая ЧСС. У женщин можно отметить абстиненцию в течение последнего года. Триада факторов - курение, ФП и высокое АД имеет довольно большую доказательную базу, и их коррекция включена в большинство рекомендаций по профилактике инсульта [19].

Смертность от других не ССЗ причин ранее не была представлена подробно в наших работах, но, учитывая общность факторов риска, мы проанализировали эту причину наряду с другими. Общим фактором для мужчин и женщин является только курение. Причем, дополнительно для мужчин самыми статистически значимыми являются низкий ИМТ и высокий пульс, значимы также низкий ХС и повышенное АД. На пределе значимости чрезмерное потребление алкоголя. У женщин к курению в модель добавляется высокое АД. Учитывая, что в не ССЗ смертности сосредоточены такие причины, как рак разной локализации, а также внешние причины, понятна полученная комбинация факторов: чрезмерное потребление алкоголя и курение в сочетании с низкой массой тела, низким ХС и высокой ЧСС, более характерные для мужчин. Известно, что внешние причины в 2-3 раза реже регистрируются у женщин, что делает более ясной не слишком выраженную зависимость факторов с данной причиной в женской когорте.

Вызывают интерес многочисленные ассоциации между высокой ЧСС и практически каждой из анализируемых причин смертности в мужской когорте и для общей смертности у женщин. Полученные в данном исследовании результаты позволяют высказать предположение, что у мужчин высокая чСС может служить не только фактором риска смертности от сердечно-сосудистых причин, но и маркером состояния здоровья, поскольку из- 
excessive alcohol consumption and smoking combined with low body weight, low TC and high heart rate which are more typical of men. External causes of death are known to 2-3-time more rarely be registered in women than in men, this explains not quite significant dependence of RF with non-CVD mortality rates in the women's cohort.

Associations between high heart rate and practically every of the cause-specific death in the men's cohort and all-cause mortality in the women are quite interesting. The received in our study data can testify that high heart rate is not only the risk factor for cardiovascular death but also the marker of health state in men as associations between heart rate and $\mathrm{BP}$, heart rate and smoking, alcohol consumption and obesity are known $[20,21]$. Unfortunately we can not make the similar conclusion for the women's cohort which is possibly explained by insufficient sample size and, respectively, insufficient number of cases even for 20year follow-up period.

Presence of the CHD diagnosis dramatically increased death risk in men what could be consider as health care failure $[22,23]$.

\section{Conclusion}

Considerably larger number of unfavorable risk factors were in the men's cohort, which significantly increased risk for death from any cause.

Statistically more pronounced relation between RF and mortality rates in men as compared to women was found, especially note that mortality rates were followed up for 10 years in men and 20 years in women.

It is obvious that successful prevention focused on risk factors must be gender-based. When summarize the received data one can conclude that men more often die of CHD and external causes while women - of stroke. Consequently, unacceptably low adherence to primary and secondary prevention of noncommunicable diseases in population and professional medical community becomes obvious.

Limitations. Small sample size and insufficient number of cases in the women's cohort; various follow-up period for men and women, confounders might interfere in the last case.

Disclosures. All authors have not disclosed potential conflicts of interest regarding the content of this paper. вестны ассоциации между ЧСС и АД, ЧСС и курением, потреблением алкоголя и ожирением $[20,21]$. Однако мы не можем сделать аналогичный вывод для женской когорты, что, вероятнее всего, объясняется недостаточным объемом выборки и, соответственно, недостаточным количеством случаев, даже при 20-летнем периоде наблюдения.

Наличие диагноза ИБС у мужчин в нашем исследовании драматически увеличивает риск смерти у мужчин от этой причины, что заставляет задуматься о доступности и качестве медицинской помощи этим больным $[22,23]$.

\section{Заключение}

Выявленные гендерные различия ассоциаций факторов риска с причинами смерти свидетельствуют: а) о значительно большем количестве прогностически неблагоприятных факторов риска, одновременное включение в модель которых существенно увеличивает в мужской когорте риск смертности по любой причине; б) о статистически значимо более выраженной силе связи между факторами и смертностью у мужчин по сравнению с женщинами, особенно, если учитывать, что смертность наблюдалась среди мужчин в течение 10 лет, а среди женщин - 20 лет.

Становится очевидным, что успешная профилактика, направленная на факторы риска, должна быть более мощной и, безусловно, гендерной. Если суммировать полученные данные, то можно сказать, что мужчины чаще умирают от ИБС и внешних причин, а женщины от инсульта. Факторы риска, ассоциирующиеся с этими причинами, подтверждают этот вывод.

Ограничения. Малый объем выборки и количество случаев в женской когорте, различный период наблюдения для мужчин и женщин (для последних более возможно действие конфаундеров) следует учитывать при интерпретации результатов.

Конфликт интересов. Все авторы заявляют об отсутствии потенциального конфликта интересов, требующего раскрытия в данной статье. 


\section{References / Литература}

1. Shkolnikov V., Andreev E., McKee M., Leon D.A. Components and possible determinants of the decrease in Russian mortality in 2004-2010. Demographic Research. 2013;28(32):917-50. [cited by Feb 20] Available from: http://www.demographic-research.org/Volumes/Vol28/32/. doi:10.4054/DemRes.2013.28.32

2. Yusuf S., Hawken S., Ounpuu S., et al. Effect of potentially modifiable risk factors associated with myocardial infarction in 52 countries (the INTERHEART study): case-control study. Lancet. 2004;364(9438):937-52. doi:10.1016/50140-6736(04)17018-9

3. Men T., Brennan P., Boffetta P., Zaridze D. Russian mortality trends for 1991-2001: analysis by cause and region. British Medical Journal. 2003;327(7421):964. doi:10.1136/bmj.327.7421.964.

4. Brainerd E., Cutler D.M. Autopsy on an Empire: Understanding Mortality in Russia and the Former Soviet Union. Journal of Economic Perspectives. 2005;19(1):107-30. doi:10.1257/ 0895330053147921

5. Razvodovsky Y.E. Alcohol Consumption and Gender Gap in All-cause Mortality in Russia. J Alcohol Drug Depend. 2017;5:271. doi:10.4172/2329-6488.1000271

6. Zaridze D., Brennan P., Boreham J., et al. Alcohol and cause-specific mortality in Russia: a retrospective case-control study of 48,557 adult deaths. Lancet. 2009;373(9682):2201-14. doi:10.1016/S0140-6736(09)61034-5.

7. Prineas R.J., Crow R., Blackburn H. The Minnesota code manual of electrocardiographic findings. John Wright-PSG; Littleton MA: 1982

8. Rose G.A., Blackburn H., Gillum R.F., Prineas R.J. Cardiovascular survey methods. Geneva: World Health Organization; 1982

9. Lorber J., Moore L.J. Gender and the Social Construction of Illness. Second Edition. Plymouth, United Kingdom: AltaMira Press; 2002.

10. Luy M., Minagava Y. Gender gaps - Life expectancy and proportion of life in poor health. Health Reports Ottawa. 2014;25(12):12-9.

11. Zarulli V., Barthold Jones J.A., Oksuzyan A., et al. Women live longer than men even during severe famines and epidemics. Proc Natl Acad Sci USA. 2018;115(4):E832-E840. doi:10.1073/pnas. 1701535115.

12. Cai J., Pajak A., Li Y., et al. Total cholesterol and mortality in China, Poland, Russia, and the US. Ann Epidemiol. 2004;14:399-408. doi:10.1016/j.annepidem.2003.10.012.

13. Bae J.M., Yang Y.J., LiZ.M., Ahn Y.O. Low Cholesterol is Associated with Mortality from Cardiovascular Diseases: A Dynamic Cohort Study in Korean Adults. Journal of Korean Medical Science. 2012:27(1):58-63. doi:10.3346/jkms.2012.27.1.58

\section{About the Authors:}

Svetlana A. Shalnova - MD, PhD, Professor, Head of Department of Epidemiology of Chronic Non-Communicable Diseases, National Medical Research Center for Preventive Medicine

Anna V. Kapustina - MD, PhD, Senior Researcher, Department of Epidemiology of Chronic Non-Communicable Diseases, National Medical Research Center for Preventive Medicine

Alexander D. Deev - PhD (Physics and Mathematics), Head of Laboratory of Biostatistics, Department of Fundamental and Applied Aspects of Obesity, National Medical Research Center for Preventive Medicine

Yulia A. Balanova - MD, PhD, Leading Researcher, Laboratory of Economic Analysis of Epidemiological Researches and Preventive Technologies, Department of Epidemiology of Chronic Non-Communicable Diseases, National Medical Research Center for Preventive Medicine
14. Romero-Corral A., Montori V.M., Somers V.K., et al. Association of bodyweight with total mortality and with cardiovascular events in coronary artery disease: a systematic review of cohort studies. Lancet. 2006;368(9536):666-78. doi:10.1016/S0140-6736(06)69251-9.

15. Pan W.H., Yeh W.T., Chen H.J., et al. The U-shaped relationship between BMl and all-cause mortality contrasts with a progressive increase in medical expenditure: a prospective cohort study. Asia Pac J Clin Nutr. 2012:21(4):577-87

16. Di Cesare M., Bentham J., Zhou B., et al. Trends in adult body-mass index in 200 countries from 1975 to 2014: a pooled analysis of 1698 population-based measurement studies with 19.2 million participants. Lancet. 2016;387(10026):1377-96. doi:10.1016/S0140-6736(16)30054-X.

17. Flegal K.M., Carroll M.D., Ogden C.L., Curtin L.R. Prevalence and Trends in Obesity Among US Adults, 1999-2008. JAMA. 2010;303(3):235-41. doi:10.1001/jama.2014.6228.

18. The Demographic Yearbook of Russia. 2015: Statistical Handbook. Moscow: Rosstat; 2015 (In Russ. [Демографический ежегодник России. 2015: Статистический сборник. М.: Росстат; 2015].

19. Meschia J.F., Bushnell C., Boden-Albala B., et al. Guidelines for the Primary Prevention of Stroke. A Statement for Healthcare Professionals from the American Heart Association/American Stroke Association. Stroke. 2014;45(12):3754-832. doi:10.1161/STR.0000000000000046.

20. Piwońska A., Piotrowski W., Broda G., et al. The relationship between resting heart rate and atherosclerosis risk factors. Kardiol Pol. 2008;66(10):1069-75

21. Papathanasiou G., Georgakopoulos D., Papageorgiou E., et al. Effects of smoking on heart rate at rest and during exercise and on heart rate recovery in young adults. Hellenic J Cardiol. 2013;54(3):168-77.

22. Tolpygina S.N., Polyanskaya Yu.N., Martsevich S.Yu. Treatment of patients with chronic ischemic heart disease in real clinical practice according to the data from PROGNOZ IBS register (part 1). Rational Pharmacotherapy in Cardiology. 2013:9(2):138-42. (In Russ.) [Толпыгина С.Н., Полянская Ю.Н., Марцевич С.Ю. Лечение пациентов с хронической ишемической болезнью сердца в реальной клинической практике по данным регистра «Прогноз ИБС» (часть 1). Рациональная Фармакотерапия в Кардиологии. 2013;9(2):138-42]. doi:10.20996/1819-6446-2013-9-2138-142

23. Boytsov S.A., Luk'yanov M.M., Yakushin S.S., et al. Cardiovascular diseases registry (RECVAZA): diagnostics, concomitant cardiovascular pathology, comorbidities and treatment in the real outpatient-polyclinic practice. Cardiovascular Therapy and Prevention. 2014;13(6):3-8. (In Russ.) [Бойцов С.А., Лукьянов М.М., Якушин С.С. и др. Регистр кардиоваскулярных заболеваний (РЕКВАЗА): диагностика, сочетанная сердечно-сосудистая патология, сопутствующие заболевания и лечение в условиях реальной амбулаторно-поликлинической практики. Кардиоваскулярная Терапия и Профилактика. 2014;13(6):3-8]. doi:10.15829/1728-8800-2014-6-3-8.

\section{Сведения об авторах:}

Шальнова Светлана Анатольевна - Д.м.н., профессор, руководитель отдела эпидемиологии хронических неинфекционных заболеваний, НМИЦ ПМ

Капустина Анна Владимировна - К.М.Н., С.Н.С., отдел эпидемиологии хронических неинфекционных заболеваний, НМИЦ ПМ

Деев Александр Дмитриевич - К.ф-м.н., руководитель лаборатории биостатистики, отдел фундаментальных и прикладных аспектов ожирения, НМИЦ ПМ

Баланова Юлия Андреевна - К.М.Н., В.Н.С., лаборатория экономического анализа эпидемиологических исследований и профилактических технологий, отдел эпидемиологии хронических неинфекционных заболеваний, НМИЦ ПМ 